\title{
La formation à l'enseignement dans une communauté enseignante. Les Frères des écoles chrétiennes du Québec 1837-1980
}

\author{
Paul Aubin \\ Chercheur associé au Centre interuniversitaire d'études québécoises, Université Laval
}

\section{RÉSUMÉ}

Fondée en France à la fin du $17^{\mathrm{e}}$ siècle pour l'instruction des garçons des classes laborieuses vivant en milieu urbain, la communauté des Frères des écoles s'implante au Québec à l'automne 1837 avec l'ouverture d'une première école. Héritière d'une longue tradition pédagogique, elle implante ici l'usage de l'enseignement simultané auquel elle joint quelques pratiques de l'enseignement mutuel déjà pratiqué au Québec. Pour contrer l'absence de toute école normale, elle commence par former ses propres recrues pour ensuite offrir le même service aux professeurs laïcs de même qu'à des communautés de sœurs enseignantes sans tradition dans l'enseignement. À partir du début de la décennie 1920, la communauté adopte les programmes des écoles normales gérées par le Département de l'instruction permettant ainsi aux religieux de se munir des mêmes diplômes d'enseignement que les instituteurs laïcs. Enfin, les frères se perfectionnent dans les rares structures universitaires dispensant des cours de pédagogie avant de fonder leur propre institut universitaire, l'Institut pédagogique Saint-Georges. Cette étude aborde, dans un premier temps, les structures dans lesquelles s'est enseignée la pédagogie chez les frères des écoles chrétiennes du Québec. La description du cadre dans lequel elle s'est enseignée devrait servir de préliminaire à une analyse du discours sur la pédagogie tel qu'exprimé dans les écrits de la communauté.

\begin{abstract}
Founded in France at the end of the seventeenth century for the education of boys from the urban working-class, the Brothers of the Christian Schools - les frères des écoles chrétiennes - opened their first school in Québec in the autumn of 1837. Drawing on a long teaching tradition, the order was responsible for introducing the use of simultaneous instruction, which they blended with certain techniques of mutual instruction already being employed in Québec. To make up for the absence of a normal school, the Brothers trained their own instructors; they would later offer the same service to lay educators and those orders of teaching sisters without a tradition of teacher instruction. Beginning in the early 1920s, the order adopted the curriculum used in the normal schools run by the Department of Education, thereby permitting religious educators to obtain the same teaching diplomas as lay instructors. Finally, after studying at the few university institutions that offered courses in pedagogy, the Brothers founded their own, l'Institut pédagogique Saint-Georges. This study examines, first
\end{abstract}


of all, the structures within which the Brothers of the Christian Schools taught educational methods. A description of this organizational framework should serve as an introduction to the discourse on pedagogy as expressed in the writings of the community.

Fondée en France à la fin du $17^{\mathrm{e}}$ siècle par le chanoine rémois Jean-Baptiste de La Salle pour l'éducation des garçons des classes laborieuses en milieu urbain, la communauté des Frères des écoles chrétiennes essaime au Québec en novembre 1837 avec un contingent de quatre frères français. Le présent texte aborde un aspect précis de la présence au Québec de cette communauté vouée à l'enseignement, soit son apport à la formation pédagogique, principalement à l'intérieur de ses propres cadres et ensuite pour d'autres communautés religieuses, voire pour des professeurs laïcs. ${ }^{1}$

L'histoire de la pédagogie québécoise attend toujours une première synthèse. Mises à part des études consacrées aux principaux théoriciens de la pédagogie, on trouve à peine quelques textes sur la façon d'enseigner telle ou telle discipline, ce qui est le propre de la pédagogie. Et, curieusement, la pléthore d'études consacrées à l'éducation par l'une ou l'autre des communautés religieuses d'enseignants escamote tout autant ce champ d'études.

Les archives de la communauté à la ville de Laval ont fourni la majeure partie des sources, tout au moins en ce qui regarde les sources manuscrites. Si elles sont riches en ce qui regarde les aspects administratifs de l'enseignement, elles sont malheureusement pauvres quant à l'appréciation de l'action pédagogique : on y retrouve peu de textes témoignant de la réflexion des administrateurs à ce sujet.

\section{La méthode pédagogique}

Quand cette communauté s'implante au Québec, le discours pédagogique des théoriciens de l'enseignement en est à ses balbutiements; à peine trouve-t-on quelques textes, notamment ceux de Joseph-François Perrault, en faveur de l'enseignement mutuel. ${ }^{2}$

De leur côté, les quatre nouveaux professeurs français, héritiers d'une longue expérience dans l'enseignement, emportent dans leurs bagages et utiliseront une méthode pédagogique - l'enseignement simultané selon lequel le professeur s'adresse à tous les élèves simultanément-coulée dans une publication de 1720, la Conduite des écoles chrétiennes, et qui, en France, est éprouvée et reconnue. Rédigée par le fondateur de la communauté en collaboration avec les frères engagés dans l'enseignement, elle n'est éditée qu'en 1720, soit un an après la mort du fondateur. ${ }^{3}$ Plusieurs fois remaniée depuis, elle demeure le condensé de la formation pédagogique dispensée aux frères, peu importe où ils oeuvrent.

Si la vision chrétienne sous-tend la démarche pédagogique prônée par le manuel de pédagogie importé de France-le titre en fait foi-, il n’en demeure pas moins qu'on y présente une vue d'ensemble de la façon d'enseigner et du fonctionnement de l'école : syllabaire, lecture, écriture, arithmétique, orthographe. Pour le fondateur de la communauté et pour ses successeurs, l'orientation chrétienne qui guide la démarche des religieux enseignants est indissociable de la profession d'instituteur 
et il le leur rappelle dans une formule typique de la littérature du XVII ${ }^{\mathrm{e}}$ siècle : " $\mathrm{Ne}$ faites point de différence entre les affaires propres de votre état [lire : la profession d'enseignant] et l'affaire de votre salut et de votre perfection [lire : l'engagement dans une communauté religieuse]. " ${ }^{4}$ Provincial $^{5}$ au Québec à la fin du XIX ${ }^{e}$ siècle, le frère Chrétien ne déroge pas de cette idée maîtresse du fondateur : "Aussi, pour ce qui nous concerne, notre vénérable fondateur place sur le même plan nos devoirs de religieux et de maître, et demande que nous apportions la même fidélité à suivre en classe notre 'Conduite des écoles' et nos 'Règles communes' en communauté. » ${ }^{6}$

Cette double finalité fait problème à certains comme le souligne le frère Sauvage, théologien : "Certains se demandent parfois s'il ne s'agit pas là d'une forme de vie hybride, inachevée [...] des catéchistes qui passent une grande partie de leur temps à enseigner les disciplines profanes [...]. " ${ }^{7}$ À ceux que rend inconfortables ce qu'ils perçoivent comme un dilemme, le même auteur rappelle la prise de conscience à l'origine de cette communauté de frères-enseignants : "L'Institut est né du 'choc existentiel' qui a secoué Jean-Baptiste de La Salle, lorsqu'il a pris conscience qu'existait à sa porte une 'jeunesse' nombreuse que sa situation matérielle et sa condition sociale privaient de l'accès à toute école, même élémentaire. ${ }^{8} \mathrm{Un}$ des plus récents analystes de cette approche pédagogique, le frère Edgar Hengemüle, résume la problématique dans une formule lapidaire : "Ce que La Salle fonda, ce fut une Congrégation de Maîtres, non de catéchistes. " La formation que les frères québécois se donneront et qu'ils inculqueront à ceux qui suivent leur exemple inclut toutes les disciplines et non seulement le catéchisme. Nous sommes loin de la vision réductrice de l'éducation de Mgr Bourget avec lequel les frères doivent composer : « [...] l'éducation religieuse doit être l'essentiel ou le principal et l'instruction intellectuelle seulement l'accessoire $[\ldots] .{ }^{10}$

\section{La formation pédagogique dispensée par les FEC}

On peut présumer que les effectifs de la communauté arrivant de France ont au moins la formation pédagogique dispensée dans leur communauté ; de plus, ils ont tous été professeurs avant d'immigrer au Québec. Reste maintenant à former les nouvelles recrues québécoises. Lieu privilégié de la formation pédagogique, l'école normale est pratiquement inexistante à l'arrivée de ces quatre Français. ${ }^{11}$ Communauté enseignante, les Frères des écoles chrétiennes devront donc se charger de la préparation à cette profession pour leurs propres membres. Quelques chiffres donnent une idée de l'évolution de la communauté au Québec; de quatre frères en 1837, on passe à 218 en 1878, 1386 en 1946, 1469 en 1956 pour commencer ensuite le déclin : 1336 en $1965 .{ }^{12}$

\section{Formation des frères par les frères}

\section{Noviciat}

Pendant longtemps la formation initiale en pédagogie se donne au noviciat : "Le Noviciat préparatoire des Frères des Ecoles chrétiennes, à Paris, est leur école normale. " 
Cette affirmation en 1841 à Montréal ne peut venir que d'un des fondateurs français ; $;^{13}$ l'utilisation, dans la même phrase, des termes " noviciat " et " école normale ", rassure les autorités et le public en général : non seulement les immigrés ont-ils reçu une formation adéquate, mais ils sont aptes à la transmettre. Ce modèle est transposé au Québec. Premier historien de la communauté au Québec et ayant vraisemblablement connu quelques-unes des premières recrues lorsqu'il publie, en 1883, son historique, l'abbé Caisse rappelle, dans une formule juxtaposant les mêmes termes, qu'au noviciat les frères " forment, dans un cours normal leurs premières recrues. " ${ }^{14}$

Sans surprise, la formation pédagogique repose sur la Conduite des écoles chrétiennes et c'est une formation continue : l'horaire prévoit la lecture de cette bible durant le déjeuner pour les frères en exercice. ${ }^{15}$ Non seulement la lit-on, mais on doit s'y conformer ; croyant devoir modifier l'horaire prévu dans la Conduite pour l'enseignement de l'arithmétique et de l'orthographe, le supérieur de Montréal demande au supérieur à Paris une dérogation bientôt accordée. ${ }^{16}$

On s'attend bien à ce que cette formation initiale soit axée sur l'enseignement simultané - le professeur s'adresse à l'ensemble des élèves plutôt que de les voir parader un par un devant lui pour répéter à chacun les explications_-, mais cette méthode doit prendre en compte la réalité québécoise des classes à divisions multiples. Résultat d'une réflexion toujours en cours, le vade-mecum qu'est la Conduite des écoles chrétiennes importée de France a continué à évoluer depuis son édition initiale en 1720 et doit tenir compte des nouvelles expériences, notamment la méthode mutuelle qui s'est implantée en France depuis l'Angleterre au début du XIX ${ }^{\mathrm{e}}$ siècle pour ensuite être expérimentée au Québec : le professeur donne l'explication à quelques élèves qui à leur tour la répètent à un petit groupe d'élèves.

Les Frères des écoles chrétiennes sont d'abord fortement opposés au mode mutuel $^{17}$ d'autant plus qu'il avait été formellement interdit par Léon XII en $1824 .{ }^{18}$ Ce qui ne veut pas dire qu'on ne peut pas en adapter certains aspects. Les premiers arrivants de la communauté peuvent s'appuyer sur la réédition de 1837 de leur vademecum qui intègre les recommandations d'un comité consultatif de 1834. Après avoir rappelé, en préface, les différents modes d'enseignement_-individuel, simultané, mutuel - le présentateur affiche clairement les couleurs de la communauté : "La méthode simultanée-mutuelle joignant aux avantages de la méthode purement simultanée, celui d'occuper plus sûrement et plus utilement les élèves, peut avoir de très-bons [sic] résultats; c'est le mode qu'indique la présente Conduite pour toutes les leçons qui en sont susceptibles. " ${ }^{19}$

Cette nouvelle mouture de la Conduite traite de disciplines absentes de la version initiale comme l'histoire, la géographie, le dessin linéaire. Ces disciplines nouvelles — tel le dessin — montrent que la pédagogie des frères de France évolue en offrant des formations utilitaires et leurs confrères québécois ne seront pas en reste ; qu'on pense à la l'enseignement de la comptabilité dont ils deviendront les spécialistes. Si on ne rejette pas d'emblée une nouvelle méthode, on l'adapte tout en maintenant que le contact professeur-élèves est essentiel, ce qui suppose que le recours au monitorat est étroitement encadré : "L'aide à des camarades moins avancés donnait lieu parfois à la désignation de responsables qui apparaissaient comme 
des moniteurs. ${ }^{20}$ Contrairement au mode mutuel, le moniteur, selon la Conduite, n'enseigne pas à l'élève de nouvelles notions, mais l'assiste dans la compréhension de notions déjà expliquées par le professeur.

La différence essentielle entre l'Enseignement Mutuel et l'Enseignement Simultané porte sur la place et le rôle du Maître. Par son organisation même — des d'élèves confiés à des Élève s Moniteurs - l'Enseignement Mutuel éloigne ipso facto le Maître des écoliers. Ce que Jean-Baptiste de La Salle et les Frères n'acceptèrent jamais. Au contraire. Ils avaient la conviction que l'essentiel du processus éducatif dépend de cette présence, proximité, connaissance personnalisée approfondie du Maître auprès de ses élèves. ${ }^{21}$

Pour les contemporains, à commencer par le surintendant Jean-Baptiste Meilleur, cette distinction n'est pas toujours claire : "L'enseignement simultané est pratiqué dans les hautes classes des écoles tenues par ces Frères, et, dans les basses classes, ils suivent l'enseignement mutuel que Lancaster a exploité. Cependant, ils réunissent ces deux méthodes, leur enseignement est souvent mixte. ${ }^{22}$ Pour Paul de Cazes, secrétaire du Département de l'instruction publique en 1905, cette ambiguïté a été levée, même dans les classes à divisions multiples. "C'est toujours le mâ̂tre qui enseigne successivement aux différents groupes. Les moniteurs ne peuvent jamais être chargés de montrer du nouveau. ${ }^{23}$

Il semble bien que le système pédagogique promu et pratiqué par les frères fasse l'unanimité, tout au moins au début, à commencer par Mgr Lartigue comme en témoigne son cri du cœur dès 1838 : "Ah ! Si tous vos enfants pouvaient être instruits, dès leurs tendres années, par les Frères des Ecoles chrétiennes [...]. » ${ }^{24}$ Faut-il y voir une preuve indirecte de l'approbation des autorités civiles au système des frères dans le fait que la loi de 1841, après avoir rappelé que les instituteurs doivent être sujets britanniques, exempte nommément les Frères des écoles chrétiennes de cette obligation ? Bien au fait de l'univers scolaire puisqu'on recourt à lui pour rédiger la loi de 1845, Augustin-Norbert Morin, dans une conférence prononcée la même année, accorde à la communauté un satisfecit : "La meilleure méthode, que je crois être composée de celle individuelle et simultanée, doit être celle des frères des écoles chrétiennes [...] ${ }^{25}$ Ancien journaliste devenu haut fonctionnaire après qu'on l'eut pressenti pour occuper le poste nouvellement créé de surintendant de l'Instruction publique, Étienne Parent, dans une conférence devant les membres de l'Institut canadien en 1848, estime que les frères sont "initiés aux secrets, aux méthodes perfectionnées d'un enseignement solide et raisonné [...]. " ${ }^{26}$ Élu député en 1851, Louis-Victor Sicotte est nommé " président d'une commission d'enquête sur l'éducation dans le Bas-Canada ". L'enquêteur inclut dans son rapport, et donc fait siens, quelques témoignages dont celui qui a rapport aux frères : "L'expérience a démontré que ce mode [simultané et mutuel] est le meilleur. C'est celui suivi par les Frères des Écoles Chrétiennes et qui donne en partie à leur enseignement, une supériorité incontestable. $"^{27}$

Ce concert de louanges laisse place à l'expression de desiderata. Une première 
friction apparaît en 1852 quand les commissaires de la ville de Québec exigent de " déterminer le cours d'études à suivre et les livres à employer dans l'enseignement ": devant les protestations des frères, les commissaires font marche arrière laissant à la communauté toute latitude dans le domaine pédagogique. ${ }^{28} \mathrm{La}$ condamnation la plus sévère vient de l'intérieur. Le frère provincial Réticius, français, porte un jugement très sévère sur ses confrères : les classes sont généralement " fort médiocres " et quant aux manuels scolaires, ils sont ce " qu'étaient, il y a 20 ans, les classiques français". En fait - et il n'y va pas de main morte — " la Science pédagogique est nulle. " ${ }^{29}$ Exagération sans doute ! Le supérieur local, dépêché depuis Paris en 1880, compare deux réalités différentes : ses confrères de France ont deux siècles d'expérience dans l'enseignement et il faut laisser le temps à la branche québécoise, toute nouvelle, de créer son propre modèle.

Appliquant leur propre méthode pédagogique, les Frères des écoles chrétiennes enseignent, pour la plupart, dans des écoles relevant des commissions scolaires et sont, de ce fait, soumis à la réglementation du Département de l'instruction publique qui inclut la pratique de l'inspection. Usage que les frères connaissent ; en 1848 ils désignent un des leurs comme inspecteur de leurs écoles de l'est de Montréal. ${ }^{30}$ Mais qu'en est-il quand les inspecteurs du DIP, dont un des mandats est de s'enquérir de la façon d'enseigner, se présentent dans leurs écoles ? Lorsque la Commission des écoles catholiques de Montréal crée le poste d'inspecteur pour ses écoles, elle essuie un refus de la part des frères ; il semble bien que ce soit beaucoup plus une réaction dictée par un réflexe d'ultramontanisme que pour des raisons d'ordre pédagogique. ${ }^{31}$ Frottements qui semblent rapidement s'estomper ; on ne relève, de la part de la communauté, aucune réaction négative à la suite de la loi de 1888 qui inclut, dans les tâches de l'inspecteur, celle de visiter " les écoles publiques établies dans chaque municipalité, soit dans une ville soit dans une campagne [...]. »

De façon générale, les frères ne semblent pas faire bande à part dans le système scolaire et ont souvent recours aux autorités laïques. Au directeur de l'école Plessis de Montréal qui demande, en 1906, deux exemplaires du Manuel de l'instituteur catholique rédigé par le secrétaire du Département de l'instruction publique, Paul de Cazes, le surintendant répond par l'envoi de douze exemplaires : les frères ne se voient pas en marge des directives faites pour l'ensemble du corps enseignant.

Pédagogie basée initialement sur un manuel français, importée et enseignée par des Français à partir de 1837, adaptée aux besoins locaux en devenant simultanée-mutuelle, elle garde toujours ses racines d'outre-mer et les renforce à l'occasion de l'arrivée massive de Français — plus de 200 — qui s'exilent à la suite des lois combistes de 1904. On les retrouve bientôt à la tête des maisons où l'on forme les nouveaux professeurs. Français, certes, mais qui doivent apprendre à composer avec les conditions locales. On leur organise un stage de six mois pour, suivant le témoignage de l'un d'entre eux, " nous mettre au courant des programmes et des méthodes en usage dans notre patrie d'adoption. $»^{32}$

\section{Scolasticat}

Prévue initialement pour être dispensée au noviciat, cette formule de formation pédagogique s'avère bientôt inadéquate d'autant plus que le noviciat est parfois écourté, 
surtout dans les premiers temps. Les effectifs s'accroissant régulièrement, la communauté peut enfin ajouter, à l'année du noviciat, une période de formation correspondant aux écoles normales : le scolasticat est enfin inauguré le 24 août $1887,{ }^{33}$ distinguant deux types de formation comme le formule Rumilly : " Le noviciat est plus spécialement consacré aux exercices religieux, et le scolasticat aux études pédagogiques. ${ }^{34}$ Aux cours théoriques pour une école "lasallienne guidée par les principes de la Conduite" s'ajoutent des leçons pratiques ; chacun des scolastiques s'exerce " en faisant à tour de rôle la classe aux confrères, voire à un groupe d'élèves de l'école voisine $[\ldots] . »^{35}$

Que la formation pédagogique se donne au noviciat en même temps que la formation religieuse ou qu'on la dispense dans une formule qui lui soit spécifiquement consacrée, il n'en demeure pas moins qu'elle se donne en vase clos avec ses propres programmes sans aucun lien formel avec ceux des écoles normales. Au début de la décennie 1920, les communautés commencent à réfléchir sur l'opportunité d'aligner les programmes de leurs scolasticats sur celui des écoles normales, ne serait-ce que pour décrocher les mêmes diplômes.

Les communautés, dont celle qui nous occupe, acceptent alors d'harmoniser leur vision du fonctionnement de leurs scolasticats avec celui prôné dans les écoles normales et le programme de ces dernières est approuvé par les autorités locales : les directoires de communautés comme la Conduite, écrits en France, passent donc au second rang. De son côté, le Conseil de l'instruction publique s'assure de l'unification des programmes dans toutes les maisons de formation de professeurs dont la majorité, les scolasticats, ne lui coûtent pas un sou.

Ce modus vivendi fonctionne pendant une trentaine d'années, chaque communauté assurant la formation de ses sujets dans le cadre des écoles normales et forçant ainsi la multiplication de services identiques d'une communauté à l'autre. À partir de 1950, elles réfléchissent à la possibilité de concentrer leurs effectifs quant à la formation pédagogique. En 1965 voient le jour le Scolasticat central de Montréal et l'école normale Notre-Dame de Foy en banlieue de Québec : un édifice commun pour les cours de pédagogie est entouré de pavillons propres à chacune des communautés qui décident de faire équipe. Une demi-douzaine de communautés d'hommes dont les Frères des écoles chrétiennes se joignent au projet pour un temps très court : l'un et l'autre cesseront leurs activités de formation pédagogique pour devenir des cégeps privés à la fin des années 1960 lors de l'intégration des scolasticats aux facultés d'éducation des universités suivant la recommandation formelle du rapport Parent s'appliquant aux écoles normales tout aussi bien qu'aux scolasticats.

\section{Appréciation de la formation pédagogique donnée aux frères}

Les références aux Frères des écoles chrétiennes, particulièrement dans la littérature pédagogique, manifestent l'intérêt qu'on porte toujours à leur façon d'enseigner et suggèrent de s'en inspirer. Dans un résumé de l'histoire de la pédagogie publié dans L'enseignement primaire et visiblement repris d'une publication française, on affirme que le système mis au point par de La Salle, " ordinairement si mal apprécié, [...] a eu une grande influence sur l'école : son ouvrage, la conduite des écoles, toujours 
ponctuellement suivi par les Frères, renferme bien des points à imiter. " ${ }^{36}$ Alors qu'au début du XX $\mathrm{XX}^{\mathrm{e}}$ siècle s'affrontent deux écoles d'enseignement de la lecture-les tenants de la méthode syllabique et les précurseurs de ce qu'on appellera la méthode phonique — , on fournit aux professeurs l'extrait d'un livre de pédagogie des frères de France en faveur de la seconde. ${ }^{37}$ Autre façon de manifester tout au moins de l'intérêt à la pratique pédagogique de la communauté : en 1908, le surintendant Pierre Boucher de La Bruère demande au provincial québécois qu'on lui envoie deux exemplaires du Manuel du catéchiste et s'enquiert si le troisième volume des Éléments de la pédagogie ${ }^{38}$ a paru. ${ }^{39} \mathrm{Ce}$ Manuel du catéchiste semble avoir connu une longue carrière au Québec : L'enseignement primaire en reproduit un extrait en $1935 .{ }^{40}$

L'influence des frères sur la pédagogie québécoise continue à se faire sentir hors de leurs propres structures, et parfois de façon anonyme. Durant l'année 1917, L'enseignement primaire publie une série d'articles sur l'enseignement de la géographie attribués à Edmond Gabriel dont on dit qu'ils sont extraits d'un Manuel de pédagogie publié chez Mame de Tours et qu'on peut se le procurer chez Beauchemin de Montréal. ${ }^{41}$ Il s'agit en fait du frère Gabriel-Marie, supérieur général de la communauté au moment de la publication. Dans le climat de laïcisation qui continue à prévaloir en France, on préfere utiliser un nom de plume plutôt que les initiales qui auraient desservi la vente ; prolifique auteur de manuels scolaires en France, il s'identifiait habituellement par la formule "F.G.-M. ». Ce manuel est inclus dans la liste des lectures que doivent faire les étudiants de l'Institut pédagogique Saint-Georges en $1937 .{ }^{42}$

Les modèles lasalliens européens ne sont pas uniquement français ; à preuve cet éloge posthume du frère Alexis, Belge décédé en 1910 et qui avait acquis une " renommée mondiale " dans l'enseignement de la géographie. ${ }^{43}$ La renommée de ce pédagogue était telle qu'en 1904 le Conseil de l'instruction publique avait envisagé de lui passer une commande pour dessiner une carte du Canada pour les écoles québécoises, projet qui, semble-t-il, n'eut pas de suite. ${ }^{44}$

\section{Brevets}

Qui dit formation dit aussi sanction officielle de la formation. Enseignant pour la majorité dans des écoles publiques relevant des commissions scolaires, les frères sont-ils diplômés, et, dans l'affirmative, quels diplômes sanctionnent leur activité professionnelle d'enseignants ? Rappelons qu'au moment de l'implantation de la communauté française au Québec, l'encadrement de la formation pédagogique est pratiquement inexistant. Tentant de combler ce vide, le gouvernement fait voter en 1846 une loi pour créer un " bureau d'examinateurs [...] pour examiner les instituteurs et leur délivrer ou refuser à chacun, suivant le cas, un brevet, ou certificat de capacité, après examen [...] ; " l'examen en question vise essentiellement à s'assurer que le candidat connaît au moins les notions qu'il doit enseigner et ne peut, en aucun cas, évaluer la compétence à transmettre ces connaissances. On n'est donc pas surpris de voir que, selon cette même loi, toute " personne faisant partie d'un corps religieux institué pour fin d'éducation »-il ne pouvait s'agir que des seuls Frères des écoles chrétiennes pour les communautés masculines - est exemptée de subir cet examen 
imposé aux seuls futurs enseignants des écoles publiques. Selon le surintendant JeanBaptiste Meilleur cité par Dufour, ces personnes « doivent posséder nécessairement toutes les qualifications requises pour l'enseignement. " ${ }^{45}$ Labarrère-Paulé voit dans cette exemption une reconnaissance implicite de la capacité de ces professeurs. ${ }^{46}$

On comprend que, dans ces circonstances, les autorités québécoises n’encouragent pas les frères à se munir d'un tel certificat. Il n'en demeure pas moins que cette exemption est battue en brèche y compris par certains membres du Conseil de l'instruction publique, dont L.-R. Masson, qui présente, en 1893, une motion à l'effet d'obliger les religieux à se munir des mêmes diplômes que les instituteurs laïcs. Aussitôt, le frère Flamien, provincial, envoie une circulaire à tous les évêques pour exprimer son opposition ; en clair, toujours le même argument : la lettre d'obédience suffit ! ${ }^{47}$

Ce qui ne veut pas dire que tous s'abstiennent de se présenter aux examens. Selon Rumilly, «des Frères canadiens ont pris, individuellement et discrètement, des brevets d'enseignement primaire supérieur. ${ }^{48}$ D'autant plus que les autorités supérieures de la communauté en France commencent à afficher une attitude contraire. ${ }^{49}$ Il n'en demeure pas moins que les autorités québécoises de la communauté continuent à garder leurs distances de la course aux diplômes d'autant plus que des instances du monde scolaire, comme Thomas Chapais ${ }^{50}$ ou l'abbé Émile Chartier, ${ }^{51}$ les approuvent.

Combat d'arrière-garde, car l'attitude vis-à-vis de cette exemption est de moins en moins partagée, car de moins en moins justifiée. Publiant le rapport d'une visite en Europe pour s'enquérir du fonctionnement du système scolaire, C.-J. Magnan — éditeur de la revue L'enseignant primaire et très près du cercle des évêques - mentionne incidemment que les religieux en Belgique sont obligés de se munir des certificats comme l'ensemble du corps professoral. ${ }^{52}$ L'attitude change également dans les communautés comme le souligne, en 1927, le rapport d'une commission d'enquête sur les écoles de Montréal : "La loi autorise les religieux à enseigner même s'ils ne sont pas pourvus de diplômes. C'est une chose que des circonstances particulières ont justifiée. Mais depuis plusieurs années, les communautés enseignantes ont incité leurs sujets à subir les examens requis des instituteurs laïques. " ${ }^{53}$ Anticipant ces changements, les frères prennent les devants : dès 1927 on inaugure « les cours de pédagogie au petit noviciat pour la préparation aux brevets élémentaire et supérieur [...]. " ${ }^{54}$ Deux ans plus tard, selon un document émanant des autorités des diverses communautés, ces dernières « croiraient avantageux d'organiser d'une manière approuvée par le Conseil de l'Instruction publique leurs Scolasticats en Écoles normales [...]. Il semble qu'un diplôme d'École normale, en attestant la culture générale et les aptitudes pédagogiques des porteurs, serait en même temps un moyen d'émulation pour jeunes religieux et un témoignage aux yeux des commissions scolaires. " 15

Dès que les frères, au début de la décennie 1930, affilient leurs scolasticats au réseau des écoles normales, ils n'ont plus d'autre choix que de suivre les mêmes programmes et de se présenter aux mêmes examens. Ce qu'on constate à l'occasion des fêtes commémorant le centenaire de leur arrivée en terre québécoise : "Les études du jeune religieux sont orientées vers l'obtention des parchemins officiels de l'État : brevet élémentaire, brevet complémentaire, brevet supérieur. Aucun sujet ne sera appliqué au labeur de l'enseignement s'il n'est porteur de l'un ou l'autre de ces 
diplômes. " ${ }^{56}$ Dans une lettre ouverte adressée aux frères déjà en exercice et publiée dans la revue officielle de la communauté, le frère Pius, sous-directeur du scolasticat, y va d'un vibrant plaidoyer en vue de l'acquisition des diplômes. ${ }^{57}$

\section{Bureau des études}

Si la formation initiale en pédagogie se donne d'abord au noviciat et ensuite au scolasticat, elle doit, espère-t-on, se continuer par la suite. À cet effet, l'institut crée un organisme, le Bureau des études, chargé de coordonner et planifier les études pour les frères qui désirent approfondir leurs connaissances alors qu'ils sont déjà sur le marché du travail. Le bureau est effectivement mis sur pied en 1936 et en deux entités, puisque le territoire administré par les Frères des écoles chrétiennes est scindé en deux districts autonomes depuis 1927. Dès leur création, chacun des deux bureaux des études lance une revue destinée aux frères : Les études pour Montréal et La voix du travail pour Québec $;^{58}$ les titres sont significatifs.

À partir de 1937, le bureau des études de Montréal planifie et gère les cours d'été donnés à la propriété de la communauté à Val-Morin ; certains étés, on y retrouve plus d'une centaine d'étudiants répartis soit dans des cours propres à une discipline soit dans des cours de pédagogie. ${ }^{59}$

L'édition de nouveaux manuels nécessite une mise à jour des connaissances chez le professeur et une adaptation à de nouvelles avenues en pédagogie. Avec l'arrivée de la série belge Témoins du Christ approuvée pour les cours de religion en 1952, de nombreux « maîtres de religion " sentent le «besoin d'approfondissement doctrinal en sciences religieuses ". Pour répondre à cette demande, les frères organisent durant l'été 1958 une série de cours donnés à Val-Morin. C'est une session intercommunautaire accueillant des frères de neuf communautés différentes ; des 119 étudiants, "tous [...] professeurs au secondaire ", 23 sont des FEC. ${ }^{60}$

\section{Formation des sœurs par les frères}

Cas rares, mais qui ont valeur de symbole : certaines communautés religieuses de femmes font appel au savoir-faire des FEC pour leur initiation à la pédagogie. Lorsqu'elle fonde la communauté des Sœurs des saints noms de Jésus et de Marie pour "l'instruction de la jeunesse ", Eulalie Durocher a bien conscience que ni elle ni ses toutes premières recrues n'ont la moindre expérience du monde scolaire et elle ne veut pas " marcher au hasard ". Solution : "Les fondatrices eurent l'inspiration d'aller chercher conseil et direction chez les Frères des écoles chrétiennes. " À l'été de 1844, deux religieuses " novices " passent les mois de juillet et août à l'école Saint-Jacques. Suivant les directives de leur supérieure, " elles assisteraient aux classes, étudieraient les procédés et les moyens disciplinaires, résumeraient chaque soir ce qu'elles auraient vu dans la journée, et de leurs notes et observations, on espérait arriver à composer un ensemble de règles et d'usages que les sœurs s'approprieraient dans la mesure du possible et qui feraient foi dans leur congrégation. " ${ }^{61}$ Les Ursulines de Trois-Rivières ${ }^{62}$ et les Sœurs de la charité de Québec (sœurs grises)

${ }^{63}$ ont recours à la même expertise. 


\section{Formation des laïcs par les frères}

Non seulement les Frères des écoles chrétiennes assurent-ils la formation des leurs, mais ils contribuent à la formation des professeurs laïcs qu'ils côtoient et qui ne peuvent faire autrement que d'être imprégnés de la méthode lasallienne. À la demande de l'évêque de Montréal, ils s'engagent même, en 1842, à donner des cours aux instituteurs laïcs qui en feront la demande. ${ }^{64}$ On semble bien être passé de la parole aux actes si on en croit le Mémorial de Meilleur publié en 1860. ${ }^{65}$ Rappelant que les frères, en France, ont formé des instituteurs laïcs dans leurs écoles, le frère Réticius se permet une comparaison : "Ainsi en fut-il en Canada jusqu'à la création des Ecoles normales, en 1857, c'est-à-dire pendant un laps de vingt ans. "66

Aux instituteurs laïques formés à l'école des frères, il convient d'ajouter ceux, nombreux, qui enseignent dans leurs écoles quand la communauté manque d'effectifs pour remplir les postes prévus dans les ententes avec les différentes commissions scolaires. Suivant Labarrère-Paulé, la " présence [des frères] est stimulante pour les instituteurs laïques. " ${ }^{67}$ Il faut aussi prendre en compte ceux qui, formés à l'intérieur des cadres de la communauté, ont décidé, souvent après quelques années d'enseignement, de se retirer. ${ }^{68}$

Enfin, la pédagogie importée de France par des Français peut rejoindre les professeurs anglophones du Québec par la publication à Montréal en 1850 d'une version anglaise de la Conduite des écoles chrétiennes qui peut aussi servir aux frères anglophones formés à Montréal pour les maisons des États-Unis. ${ }^{69}$

Peut-on envisager un transfert dans l'autre sens, à savoir que la pédagogie des instituteurs laïques aurait influencé celle des frères ? Une des premières recrues, F.-X. Goulet, a déjà été professeur avant de joindre, pour une courte période, la communauté. ${ }^{70}$ Cas sans aucun doute rare, mais non unique, tel ce frère Placidius of Sicilia : "Né au Cap Santé de Portneuf, en 1849, il entra au noviciat, en 1869, après avoir fait un cours brillant à l'école normale de Québec. " ${ }^{71} \mathrm{Ou}$ encore Norbert Thibault connu sous le nom de frère Oliver Julian de 1872 à 1875 ; recrue prestigieuse d'un ancien élève de l'école normale Laval et pédagogue très en vue lorsqu'il se joint, brièvement, à la communauté. ${ }^{72}$

\section{La formation pédagogique en milieu universitaire}

\section{Formation universitaire à l'étranger}

Pour mieux assumer ses responsabilités, la congrégation envoie quelques-uns de ses membres poursuivre des études supérieures en Europe. Ainsi, durant la décennie 1930, les frères Léo et Chrysostome s'inscrivent à l'école de pédagogie de l'Université catholique de Louvain et, après quatre années, reviennent tous deux avec le titre de docteur en sciences pédagogiques et psychologiques. ${ }^{73}$ Longtemps professeur à la fois au scolasticat et à l'Institut pédagogique Saint-Georges, le frère Ephrem soutient, en 1963, sa thèse à l'Université de Fribourg jugée summa cum laude et intitulée : Les corrélations en pédagogie et en psychologie. ${ }^{74}$ Pour le frère Jules Cantin, ce sera l'Université 
de Genève où il soutient une thèse traitant de l'apprentissage du vocabulaire et de l'orthographe. ${ }^{75}$

\section{Formation universitaire au Québec}

\section{Enseignement moderne et pédagogie}

Offerts par l'Université Laval à Montréal en $1910^{76}$ à la demande de la Commission des écoles catholiques de Montréal, ${ }^{77}$ ces cours s'adressent particulièrement aux religieux qui, n'ayant pas suivi le cours classique, ne peuvent avoir accès à l'université.

Pour l'abbé Chartier dont il est fait mention plus haut et qui enseigne dans cette université, on veut ainsi préparer les religieux qui, de plus en plus nombreux, enseignent au secondaire. On offre une programmation de type culture générale en regard de disciplines nouvelles pour le cours secondaire ou à peine effleurées au cours primaire et donc plus ou moins ignorées dans les scolasticats où on formait une clientèle destinée majoritairement au primaire, tout au moins au début ; en complément, on prévoit ajouter des cours de pédagogie. En fait, la part réservée à l'enseignement de la pédagogie est on ne peut plus restreinte dès le début de l'Enseignement moderne et pédagogie et le nom même de cette discipline disparaît ensuite des programmes de cette école de pédagogie universitaire ; compte tenu de la clientèle visée - des religieux qui en plus d'avoir eu des cours de pédagogie dans leurs communautés respectives sont des professeurs en exercice - , on peut penser qu'on les estimait compétents dans l'art d'enseigner.

On y trouve au moins un frère dans le corps professoral : le frère Martinus donne le cours "Dessin - Application de cet enseignement dans les collèges" à l'été 1917 ; professeur au collège commercial des frères à Montréal, le Mont-Saint-Louis, c'est dans cette institution que se donnent les cours du programme Enseignement moderne et pédagogie durant la saison estivale de $1917 .^{78}$

De 1919 à 1923, quatorze frères des écoles chrétiennes décrochent ce diplôme, la plupart avec grande distinction, et vingt-deux en 1930-1931 ; une liste cumulative non datée, incomplète et émanant du bureau des études dresse une liste de 32 frères qui ont réussi les examens de cet organisme. ${ }^{79}$ Encore faut-il se demander quelle formation les frères vont y chercher. Sur les vingt-trois disciplines offertes selon le programme pour l'année 1918-1919 et qui n'évolue pas par la suite, une seule aborde la pédagogie, et encore est-ce l'histoire de la pédagogie, les vingt-deux autres étant dévolues aux disciplines que les frères doivent enseigner dans les institutions de niveau secondaire. ${ }^{80}$

\section{Institut pédagogique Saint-Georges ${ }^{81}$}

La communauté des Frères des écoles chrétiennes a une longue expérience dans la création et l'administration de structures universitaires vouées à la pédagogie, notamment en Belgique, aux États-Unis et en Amérique latine ; le Québec ne fait pas exception.

Les sœurs de la Congrégation de Notre-Dame avaient créé un institut pédagogique destiné aux institutrices en 1926. Les Frères des écoles chrétiennes emboîtent 
le pas en 1929 avec la création de l'Institut pédagogique Saint-Georges à la demande expresse de l'évêque auxiliaire de Montréal qui est aussi chancelier de l'Université de Montréal, Mgr Georges Gauthier. Sitôt approuvé par le comité catholique du Conseil de l'instruction publique, ${ }^{82}$ l'IPSG ambitionne d'être reconnu comme " une école universitaire de pédagogie et non une 'école normale' fut-elle supérieure. " Logé temporairement au Mont-Saint-Louis avant d'occuper des locaux à l'Université de Montréal à laquelle il est affilié dès sa création, cet "institut est une école supérieure de pédagogie ; il est comme le prolongement et le couronnement de l'Institut de l'enseignement moderne et de pédagogie. " ${ }^{83}$ On notera l'ambiguïté des termes si on compare cette formule à celle indiquée quelques lignes plus haut : l'IPSG prend ses distances de l'école normale traditionnelle où, à côté de l'apprentissage des notions de base en pédagogie, on est obligé de consacrer beaucoup de temps à la maîtrise des connaissances que le futur professeur doit enseigner. ${ }^{84}$ Très tôt, l'IPSG dispense son enseignement tout autant aux instituteurs religieux qu'aux laïcs. ${ }^{85}$ En 1935, les étudiants peuvent décrocher un « Diplôme de pédagogie supérieure, [un] Diplôme de psychologie pédagogique et de psychologie expérimentale [et des] grades universitaires de bachelier, de licencié et de docteur en pédagogie. ${ }^{86}$ En plus des cours réguliers, l'IPSG organise des conférences pour auditeurs libres auxquels sont expressément invités les frères. ${ }^{87}$ La participation des frères dépasse la seule mise en œuvre de l'Institut. Ainsi, en 1935, des douze professeurs, cinq sont des leurs. ${ }^{88}$

La formation dispensée à l'IPSG semble appréciée par les instances administratives : depuis le Département de l'instruction publique qui « reconnaît cette licence comme donnant droit d'enseigner dans les écoles normales " jusqu'au ministère du Bien-être social et de la Jeunesse qui « envoie, chaque année, une cinquantaine de professeurs des écoles spécialisées et [...] paye leur cours en entier. " Ailleurs, ce sont des commissions scolaires, telles celles de Verdun et de Trois-Rivières, qui " accordent des gratifications " aux diplômés de l'IPSG. ${ }^{89}$ Autre manifestation d'intérêt : en 1955, les provinciaux des différentes communautés enseignantes " prient l'Institut St-Georges d'organiser pour les vacances prochaines des cours de Méthodologie catéchistique, et plus tard divers cours se rapportant à la religion. " ${ }^{90}$

Institution universitaire, l'IPSG, en plus de dispenser de l'enseignement, doit aussi développer une politique de recherche, ce dont témoigne la série de Bulletins qu'il produit à partir de 1939. Des quatorze communications qu'on y relève, neuf sont rédigées par des frères qui y oeuvrent. ${ }^{91}$ Un des professeurs de l'IPSG, le frère Chrysostome, semble même être devenu une vedette dans le monde de la recherche en pédagogie avec ses travaux sur les enfants exceptionnels, tant surdoués que sousdoués ; on le retrouve sur toutes les tribunes, que ce soit dans une revue médicale — "L'étude des anormaux est au programme de tous les instituts de pédagogie " — , à un congrès d'instituteurs ou dans La revue trimestrielle canadienne. ${ }^{92}$

Le statut international de l'institut des Frères des écoles chrétiennes favorise les échanges et les transferts pédagogiques d'un pays à un autre, notamment au chapitre des manuels scolaires. Bénéficiant de son appartenance à la même structure, l'IPSG connaît un rayonnement que ses fondateurs n'avaient pas envisagé. Professeur de 
psychopédagogie à l'école normale La Salle dirigée par les FEC à Canoas en banlieue de Porto Alegre (Brésil) durant la décennie 1960, le frère Henrique Justo traduit en portugais pour ses étudiants des tests produits par ses confrères de Montréal ; ${ }^{93}$ certains de ces tests y seront encore en usage alors que l'IPSG de Montréal aura cessé d'exister depuis longtemps.

Dirigé par les frères Ephrem (1929-1935), Léo (1936-1942), Luc (1942-1955) et Charles (Dufour) (1955-1964), l'IPSG est intégré à la faculté des sciences de l'éducation de l'Université de Montréal en juin 1966. ${ }^{94}$

\section{École de pédagogie et d'orientation}

Fondée en 1943 par l'Université Laval, cette institution compte, dans son corps professoral des FEC. Les noms des frères Clément (Lockquell) et Alfred (Houle) apparaissent régulièrement comme professeurs dans les annuaires de cette école à partir de 1943 ; occasionnellement on y trouve le nom du frère Marie-Hector. Le frère Maurice-Alexandre y soutient une thèse de licence dans laquelle il établit un lien entre les idées pédagogiques du fondateur de la communauté et la pratique de cette pédagogie au XX siècle. $^{95}$

\section{Société de pédagogie de Montréal}

Créée en 1938 par le frère Chrysostome de l'Institut pédagogique Saint-Georges et dont il est élu premier président, ${ }^{96}$ cette société évolue hors du cadre universitaire, mais regroupe un certain nombre d'universitaires désireux d'échanger le résultat de leurs recherches en pédagogie ou de pédagogues faisant part de leurs expériences. Elle a son siège social à l'IPSG et tient ses réunions au Mont-Saint-Louis. "La Société a pour objet : 1-, l'étude et la vulgarisation des sciences pédagogiques et psychologiques au Canada ; 2-, la poursuite des travaux de recherches scientifiques ; 3-, l'établissement de relations scientifiques entre les pédagogues et les psychologues canadiens et étrangers. " ${ }^{97}$

Le programme-souvenir du $10^{\mathrm{e}}$ anniversaire de la société présente la liste de 59 conférences qu'on y a données, dont onze par les FEC. ${ }^{98}$ Lorsqu'elle fête ses vingt-cinq ans, elle compte, parmi ses présidents à vie, le frère Charles, également du même IPSG. ${ }^{99}$ Elle comprend 38 membres inscrits pour l'année 1939-1940, dont les frères Chrysostome et Nivard-Anselme. ${ }^{100}$ Durant la décennie 1940, au moins cinq frères donnent des conférences aux membres de cette société : le frère Dominique est professeur à l'IPSG tout comme le frère Chrysostome, qui dresse un portrait de la situation de la recherche en pédagogie ; le frère Grégoire est professeur soit à l'IPSG, soit au scolasticat de la communauté à Laval-des-Rapides ; un autre, le frère Ephrem (Brisebois), est également professeur dans les deux mêmes institutions. ${ }^{101}$ Professeur à l'école secondaire Maisonneuve, le frère Denis (Champagne) donne une conférence sur la lecture : "Nous avons tout à gagner à conduire nos enfants devant la tribune du plus efficace et du plus réconfortant des maîtres, la lecture. " ${ }^{102}$ 


\section{Conclusion}

Appliquant au Québec une pédagogie importée de France, les quatre Français qui arrivent ici en 1837 n'ont d'autre choix que d'adapter un modèle éprouvé ailleurs, mais qui nécessite des ajustements locaux, ne serait-ce que pour les écoles à divisions multiples. De plus, il leur faut non seulement " discipliner ", au sens très large du terme, les élèves, mais également former une relève, car la France, après avoir fourni les matériaux initiaux, s'attend que le rejeton acquière son autonomie. La communauté québécoise doit donc se recruter et former ses recrues à l'enseignement.

Au fur et à mesure que les besoins de l'enseignement se développent et se spécialisent, les frères doivent s'y adapter; d'abord concentrés dans l'enseignement primaire, leurs effectifs envahissent le secteur secondaire. ${ }^{103}$ Leur cheminement québécois évolue dans le même sens que celui de l'ensemble de l'institut. Créé en France pour l'instruction des débutants — entendons les écoles primaires — l'institut, d'abord en France et ensuite ailleurs dans le monde, intègre bientôt à sa vision de l'enseignement les élèves qui veulent une formation plus avancée. Pour ces élèves, on offrira des cours plus utilitaires en vue d'ouvrir les portes vers un meilleur gagne-pain ; au Québec, les Frères des écoles chrétiennes investiront le champ des études commerciales : l'Académie commerciale qu'ils fondent au XIX ${ }^{\mathrm{e}}$ siècle débouchera vers la fondation de la faculté de commerce de l'Université Laval au XX ${ }^{\mathrm{e}}$ siècle. ${ }^{104}$ Quand apparaissent les premières structures universitaires pour une formation pédagogique plus poussée que celle offerte dans les écoles normales, on les y retrouve, d'abord comme étudiants et bientôt comme professeurs, avant de créer leur propre centre de formation universitaire en pédagogie.

J'ai tenté de circonscrire le cadre dans lequel la communauté s'est engagée dans la formation des futurs professeurs, d'abord ses propres membres pour ensuite élargir cette formation hors de son propre milieu.

Restent d'autres champs de recherche que j'ai volontairement escamotés. En premier lieu, le jeu des influences des intervenants dans le monde scolaire. Les grands théoriciens de l'éducation au Québec — Valade, Langevin, Rouleau/Magan/Ahern, Ross, Laurence, Vinette — ont-ils été influencés par la pédagogie lasallienne dans leurs écrits ? À l'inverse, ces écrits ont-ils influencé les frères quant à leur conception de l'enseignement et aux méthodes de la vivre en classe ? Qu'en est-il de pratique de la pédagogie quand les frères se retrouvent au front, que ce soit comme auteurs de manuels scolaires ou professeurs? Appliquent-ils les principes qu'on leur a inculqués et font-ils preuve de créativités face aux situations nouvelles ? La Conduite de 1720 ne prévoyait ni les camps de sciences ni les ciné-clubs.

Des réponses à ces questions nous aideraient à voir si la généreuse affirmation de Caporicci quant aux FEC de la période 1837-1847 peut être étendue à la période qui suit ou si elle nécessite des ajustements : "The methods developed by La Salle and used by his followers constitute the main benefit that the religious community transferred from France to Canada. ${ }^{105}$ 


\section{Notes}

1 Pour une histoire de cette communauté enseignante au Québec, la plus importante, numériquement, quant aux effectifs comme pour la production des manuels, voir les trois tomes de Nive Voisine, Les frères des écoles chrétiennes au Canada (Sainte-Foy: Anne Sigier : 1987, 1991, 1999). Pour les manuels scolaires, voir http://www.bibl. ulaval.ca/ress/manscol/ et entrer " fec » dans le champ " éditeur ». Cet article s'inspire d'une version beaucoup plus longue et descriptive parue dans la Revue numérique de recherche lassallienne. Ce texte s'avère fort différent de la version électronique, http://revista_roma.delasalle.edu.mx/numero_11/paul_aubin_11.pdf.

2 Joseph-François Perrault, Cours d'éducation élémentaire à l'usage de l'école gratuite établie dans la cité de Québec en 1821 (Québec : Nouvelle imprimerie, 1822).

3 Léon Lauraire, La Conduite. Approche contextuelle, coll. "Cahiers lasalliens " 61 (Rome : Maison Saint-Jean-Baptiste de La Salle, [2001]), 3.

4 Euvres complètes - Saint-Jean-Baptiste De La Salle (1651-1719), (Rome : Frères des écoles chrétiennes, 1993), 106.

5 On utilise le terme « directeur " pour désigner le responsable d'une " communauté » ou école. Un ensemble de " communautés " forme un " district " dont le responsable est identifié sous les termes " provincial " ou "visiteur ", cette dernière formule rappelant qu'une partie importante de sa charge consiste à visiter les frères dans les différentes " communautés "; j'utilise dans ce texte la formule " provincial ".

6 Lettre à Mgr Fabre, 15 avril 1887, Archives des FEC, chemise 503135.

7 Michel Sauvage, Catéchèse et lä̈cat-Participation des lä̈cs au ministère de la Parole et mission du Frère enseignant dans l'Église (Paris : Ligel, 1962), 1.

8 Michel Sauvage et Miguel Campos, L'espérance fragile d'un témoin - L'itinéraire du F. Michel Sauvage (1923-2001) (Rome : Frères des écoles chrétiennes, 2014), 420. Non pas qu'il n'existait pas d'écoles pour la classe populaire mais elles fonctionnaient de façon erratique et les professeurs manquaient de toute préparation. Voir Yves Poutet, «La ville de Reims et l'éducation des garçons et des filles de milieux modestes au temps de Louis XIV ", Originalité et influence de saint Jean-Baptiste de La Salle-Recueil d'études lasalliennes (I-II), coll. "Cahiers lasalliens " $\mathrm{n}^{\circ} 43$ (Rome : Frères des écoles chrétiennes, 1999) : 77-116.

9 Edgar Hengemüle, Une proposition éducative-Jean-Baptiste de La Salle (Paris : Salvator, 2012), 104.

10 Mélanges religieux, 7 mars 1845. Cité dans Marcel Lajeunesse, "Lévêque Bourget et l'instruction publique au Bas-Canada, 1840-1846» dans Marcel Lajeunesse, L'éducation au Québec (19'-20 siècles) (s.1. : Boréal express, 1971), 55. Mélanie Lanouette, sans doute emportée par l'enthousiasme avec lequel elle traite son sujet, affirme que « [...] pour Jean-Baptiste de La Salle, l'Institut des Frères des Écoles chrétiennes est principalement, sinon exclusivement voué à l'enseignement du catéchisme [...]. » dans Faire vivre ou faire connaître - Les défis de l'enseignement religieux en contexte de renouveau pédagogique - 1936-1946 (Québec : Les presses de l'Université Laval, 2002), 17-18.

11 Deux écoles normales sont prévues par la loi de 1836. Celle de Québec-école normale Laval - n'ouvrira qu'à la fin de la décennie 1850 alors que celle de Montréal_école normale Jacques-Cartier - vivote de septembre 1837 à sa fermeture en 1842. LouisPhilippe Audet et Armand Gauthier, Le système scolaire du Québec_Organisation et fonctionnement (Montréal : Beauchemin, 1967), 11-12.

12 Nive Voisine, Les frères des écoles chrétiennes au Canada (Sainte-Foy : Anne Sigier : 1987, 1991, 1999), t. $1: 255$; t. $2: 366$; t. $3: 248$.

13 "Noviciat des écoles chrétiennes ", Supplément des mélanges religieux, 4 (21 mai 1841): 18-19. Les italiques sont dans le texte.

14 J.-C. Caisse, Linstitut des Frères des écoles chrétiennes - Son origine, son but et ses oeuvres (Montréal : J. Chapleau, 1883), 85. 
15 François de Lagrave, Frère Réticius, F.E.C. - Le mandat tumultueux d'un visiteur provincial - 1880-86, thèse de M.A. (histoire), Université Laval, 1977, 34.

16 Avis et permissions concernant le Communauté des Frères de Montréal, Archives des FEC, chemise 506433.

17 Léon Lauraire, La conduite des écoles - Approche diachronique - Évolution du texte de 1706 à 1916, coll. «Cahiers lasalliens » 67 (Rome : Frère des écoles chrétiennes, 2014), $112-117$.

18 Dell Upton, «Écoles lancastériennes, citoyenneté républicaine et imagination spatiale en Amérique au début du XIX siècle ", Histoire de l'éducation, 102 (1 ${ }^{\mathrm{er}}$ mai 2004) :87108. L'enseignement mutuel disparaît aux États-Unis vers 1850.

19 Conduite des écoles chrétiennes, par le vénérable De La Salle [...], Nouvelle édition, revue, corrigée et approuvée par le chapitre général de 1837 (Paris: Tre Moronval, 1856). Je n’ai pu trouver cette édition de 1837 mais on peut supposer que l'édition de 1856, compte tenu de la formulation en page de titre, reprend le texte de 1837.

20 Léon Lauraire, La "Conduite des écoles"-Approche pédagogique, coll. "Cahiers lasalliens" n 62 (Rome : Maison Saint-Jean-Baptiste de La Salle, 2006), 222.

21 Id, La Conduite des écoles - Approche diachronique - Évolution du texte de 1706 à 1916, coll. "Cahiers lasalliens " nº 67 (Rome : Frères des écoles chrétiennes, 2014), 114.

22 Jean-Baptiste Meilleur, Mémorial de l'éducation du Bas-Canada (Montréal : J.-B. Rolland \& fils, 1860), 101.

23 Paul de Cazes, Manuel de l'instituteur catholique de la province de Québec (Montréal : Librairie Beauchemin, 1905), 22. Les italiques sont de l'auteur.

24 Mandements - Lettres pastorales - Circulaires et autres documents publiés dans le diocèse de Montréal depuis son érection jusqu’à l’année 1869-Tome premier (Montréal : J. Chapleau \& fils, 1887), 49.

25 Augustin-Norbert Morin, "De l'éducation élémentaire-Ce qu'elle est et ce qu'elle doit être » dans J. Huston, Le répertoire national ou recueil de littérature canadienne-Volume III (Montréal : J. M. Valois, 1893), 224.

26 Etienne Parent, "Considérations sur notre système d'éducation populaire, sur l'éducation en général, et les moyens législatifs d'y pourvoir » dans J. Huston, $L e$ répertoire national ou recueil de littérature canadienne - Volume IV (Montréal : J. M. Valois, 1893), 336.

27 Louis-Victor Sicotte, Rapport du comité spécial de l'assemblée législative nommé pour s'enquérir de l'état de l'éducation et du fonctionnement de la loi des écoles dans le Bas Canada (Québec : John Lovell, 1853), 31.

28 Germain (frère), "Les pionniers à Québec ", L'oeuvre d'un siècle-Les Frères des écoles chrétiennes au Canada (Montréal : Les Frères des écoles chrétiennes, 1937), 82.

29 François De Lagrave, "Le mandat tumultueux d'un visiteur provincial : le frère Réticius, f.e.c. (1880-1886" dans Nive Voisine et Jean Hamelin, Les ultramontains canadiensfrançais (Montréal : Boréal express, 1985), 243.

30 Grégoire (frère), "Les quatre fondateurs de l'institut au Canada " dans L'oeuvre d'un siècle-Les Frères des écoles chrétiennes au Canada (Montréal : Les Frères des écoles chrétiennes, 1937), 69.

31 Louis-Philippe Audet, "Épisode scolaire de la lutte ultramontaine à Montréal ", Les cahiers des dix, 39 (1974) : 18.

32 Guy Laperrière, Les congrégations religieuses - De la France au Québec - 18801914-Tome 2-Au plus fort de la tourmente - 1901-1914 (Sainte-Foy : Les presses de l'Université Laval, 1999), 456 et 460.

33 Nive Voisine, Les Frères des écoles chrétiennes au Canada - Tome II-Une ère de prospérité-1880-1946 (Sainte-Foy : Anne Sigier, 1991), 124.

Il faudrait voir sur quoi se base Thérèse Hamel pour retarder cette ouverture à 1931 : "Il faudra attendre la création des scolasticats de religieux en 1931 [...]." (Un siècle 
de formation des maîtres au Québec - 1836-1939 (Ville La Salle : HMH, 1995), 126). Au début de la décennie 1930, les scolasticats, dont certains existent depuis une quarantaine d'années, alignent leurs programmes sur celui des écoles normales de façon à s'obliger à subir les mêmes examens.

34 Robert Rumilly, Le frère Marie-Victorin et son temps (Montréal : Les Frères des écoles chrétiennes, 1949), 11. Il s'agit donc bien d'une nouvelle structure prolongeant mais non remplaçant le noviciat et on ne peut écrire que les « noviciats [.] seront peu à peu transformés en scolasticats-écoles normales [...]. » (Thérèse Hamel, Idem, 51-52).

35 Michel (frère), "La vie intellectuelle et pédagogique du frère " dans L'oeuvre d'un siècle-Les Frères des écoles chrétiennes au Canada (Montréal : Les Frères des écoles chrétiennes, 1937), 137.

36 "Histoire de la pédagogie ", L'enseignement primaire, 8, 11 (1 $1^{\text {er }}$ juin 1888) : 169. La note négative sur l'enseignement des frères peut se comprendre dans une revue française publiée en pleine montée du laïcisme en France. D'ailleurs, l'éditeur de la revue québécoise ajoute une mise en garde demandant aux lecteurs d' "être très prudents sur le choix des auteurs, et ne lire que ceux qui sont irréprochables sous le rapport des principes religieux et moraux."

37 "L'ancienne et la nouvelle méthode d'épellation comparées ", L'enseignement primaire, 27, 3 (nov. 1905) :135-136.

38 Manuel du catéchiste-Méthodologie de l'enseignement de la religion dans les écoles primaires et les pensionnats (Paris: Procure générale des frères des écoles chrétiennes, 1907) aussi Méthodologie de l'enseignement de la langue maternelle — Extrait de la IIIe partie des Éléments de pédagogie pratique (Paris : Procure générale, 1902).

39 Paul Aubin et Michel Simard, Les manuels scolaires dans la correspondance du Département de l'instruction publique-1900-1920 (Sherbrooke : Groupe de recherche sur l'édition littéraire au Québec, 2005) et un CD-ROM, entrée 1061.

40 "Une leçon de catéchisme », L'enseignement primaire, 57, 1 (sept. 1935) :19-21.

41 Edmond Gabriel, "L’enseignement de la géographie ", L'enseignement primaire, 38, 5 (janv. 1917) : 263-264 ; 6 (fév. 1917) : 344-345 ; 7 (mars 1917) : 389-390 ; 8 (av. 1917) : 463-465 ; 9 (mai 1917) : 518-519 aussi Manuel de pédagogie à l’usage des écoles catholiques (Tours: A. Mame et Paris : Poussielgue, 1909).

42 "Institut pédagogique St-Georges ", La voix du travail, 1, 16 (3 juill. 1937) :1-5.

43 "Un grand éducateur géographe - Le cher frère Alexis (1835-1910) ", L'enseignement primaire, 32, 6 (fév. 1911): 329-331.

44 Rapport du surintendant de l'instruction publique de la province de Québec pour l'année 1903-1904 (Québec: Charles Pageau, 1905), 377.

45 Andrée Dufour, Histoire de l'éducation au Québec, (s.l. : Boréal, 1997), 41.

46 André Labarrère-Paulé, Les instituteurs lä̈ques au Canada français - 1836-1900 (Québec : Les presses de l'Université Laval, 1965), 142.

47 Flamien (frère), [Circulaire du provincial de Montréal envoyée aux évêques québécois], 4 mai 1893. Archives des FEC, chemise 503177.

48 Robert Rumilly, Le frère Marie-Victorin et son temps (Montréal : Les frères des écoles chrétiennes, 1949), 11.

49 Nive Voisine, Les Frères des écoles chrétiennes au Canada-Tome II-Une ère de prospérité-1880-1946 (Sainte-Foy : Anne Sigier, 1991), 44.

50 Thomas Chapais, Les congrégations enseignantes et le brevet de capacité (Québec : Léger Brousseau, 1893), 25 et 28.

51 Émile Chartier, "L'enseignement secondaire moderne et les chers frères ", La semaine religieuse de Montréal, 64, 10 (7 sept. 1914) : 152-153.

52 C.-J. Magnan, Les écoles primaires et les écoles normales en France, en Suisse et en Belgique - Rapport présenté au Surintendant de l'Instruction publique et aux membres du Comité catholique (Québec : s.n., 1909), 306. 
53 Rapport de la Commission d'enquête sur la situation des écoles catholiques de Montréal-26 février 1926-1er décembre 1927 (s.l., s.n., n.d.), 18.

54 Léopold (frère), "Le cher frère Macarius-Léo décédé le 5 août 1960 ", Les études, 25 (nov. 1960) : 110. Précisons que les élèves ont 15 ou16 ans pour la majorité.

55 M'hammed Mellouki et François Melançon, Le corps enseignant du Québec de 1845 à 1992 - Formation et développement (Montréal : Logiques, 1995), 46.

56 Michel (frère), "La vie intellectuelle et pédagogique du frère ", L'oeuvre d'un siècle — Les Frères des écoles chrétiennes au Canada (Montréal : Les Frères des écoles chrétiennes, 1937), 137.

57 Les études, 3, 22 (sept. 1938) :7-8.

58 Nive Voisine, Les Frères des écoles chrétiennes au Canada-Tome II-Une ère de prospérité-1880-1946 (Sainte-Foy, : Anne Sigier, 1991), 263-265. En ce qui regarde l'organisation, par le bureau des études, des cours que suivent les frères en activité, je me limite aux études en pédagogie.

59 Ibid, 294.

60 Importante session d'études religieuses à Val-Morin, 23 septembre 1958. Archives des FEC, chemise 501833.

61 Mère Marie-Rose fondatrice de la congrégation des saints noms de Jésus et de Marie (Montréal : Beauchemin, 1930), 80-81.

62 Thérèse Germain, Autrefois les Ursulines de Trois-Rivières-Une école, un hôpital, un cloître (Sillery : Anne Sigier, 1997), 225.

63 Nive Voisine et Yvonne Ward, Histoire des Sours de la charité de Québec-Tome I-L'âme de la fondation Marcelle Malle (1805-1871) (Beauport : MNH, 1998), 116-117.

64 Mandements - Lettres pastorales - Circulaires et autres documents publiés dans le diocèse de Montréal depuis son érection jusqu’à l’année 1869-Tome premier (Montréal : J. Chapleau \& fils, 1887), 189-191.

65 Jean-Baptiste Meilleur, Mémorial de l'éducation du Bas-Canada (Montréal : J.-B. Rolland \& fils, 1860), 379.

66 [Frère Réticius], Aux honorables membres du comité catholique du conseil de l'instruction publique (s.l., s.n., [1884]), 15.

67 André Labarrère-Paulé, Les instituteurs lä̈ques au Canada français-1836-1900 (Québec : Les presses de l'Université Laval, 1965), 12-13.

68 [Frère Réticius], Aux honorables membres du comité catholique du conseil de l'instruction publique (s.l., s.n., [1884]), 22.

69 Government of the Christian Schools, by the Venble [sic] J.B. De La Salle, D. D., founder of the Christian Schools (Montréal : Lovell and Gibson, 1850).

70 F.S.-L. Les Frères des écoles chrétiennes au Canada, 1837-1900 (Montréal : Les Frères des écoles chrétiennes, 1921),17-18.

71 Ibid, 217.

72 Aurélien Boivin, "Thibault (Thibeault), Norbert, dit frère Oliver Julian ", Dictionnaire biographique du Canada, http://www.biographi.ca/fr/bio/thibault_norbert_11F.html consulté le 9.01.2015.

73 Nive Voisine, Les Frères des écoles chrétiennes au Canada-Tome II-Une ère de prospérité-1880-1946 (Sainte-Foy : Anne Sigier, 1991), 260. D’après les informations fournies par le Directeur des archives de l'Université catholique de Louvain, Paul Servais, le frère Léo (Mastaï Langlois) y est inscrit de 1932 à 1936 et le frère Grégoire (Omer Legault) de 1937 à 1939.

74 Gilles Beaudet, En mémoire du frère Raymond Brisebois (frère Éphrem) (Longueuil : Frères des écoles chrétiennes, 1996), 14-17.

75 Jules Cantin, L'apprentissage du vocabulaire et de l'orthographe-Un sondage pour mesurer l'efficacité de dix ans d'enseignement d'un vocabulaire au niveau élémentaire-Thèse 
présentée à la faculté de psychologie et de sciences de l'éducation de l'Université de Genève pour obtenir le grade de Docteur ès Sciences de l'éducation (Joliette : Imprimerie SaintViateur, 1978).

76 Annuaire de l'Université Laval pour l'année académique 1910-1911 (Québec : Imprimerie de l'Événement, 1910), 49.

77 «Un cours de pédagogie à l'Université Laval (Montréal) », L'enseignement primaire, 38, 6 (fév. 1917) : 383.

78 "Cours de vacances ", L'enseignement primaire, 39, 1 (sept. 1917) : 13 aussi « Propos de pédagogie ", L'enseignement primaire, 39, 1 (sept. 1917) : 4.

79 Université de Montréal - Enseignement moderne et pédagogie, Archives des FEC, chemise 503254 ; Année 1930-1931_Enseignement moderne et pédagogie (suite)—Epreuves universitaires, Archives des FEC, chemise 503979; Liste des noms des Frères qui, ayant le diplôme universitaire Lettres et Sciences de l'Institut d'Enseignement et de pédagogie [...], Archives des FEC, chemise 503268. Lapparente contradiction entre les chiffres vient du fait que certaines sources ne donnent que des résultats fragmentaires.

80 Annuaire de l'Université Laval pour l'année académique 1918-1919, Nº 62 (Québec : L'action sociale, 1918), 111-112.

81 Nive Voisine, Les Frères des écoles chrétiennes au Canada-Tome II-Une ère de prospérité-1880-1946 (Sainte-Foy: Anne Sigier, 1991), 278-291.

82 "Les Frères des écoles chrétiennes fondent un institut pédagogique à Montréal ", L'enseignement primaire, 50, 9 (mai 1929) : 561.

83 "L'Institut St-Georges est affilié ", L'enseignement primaire, 51, 5 (janv. 1930):369.

84 Chrysostome (frère), L'oeuvre de l'Institut pédagogique Saint-Georges (s.l., s.n., n.d.), Archives des FEC, chemise 500276. Plaidoyer passionné, par un des premiers artisans de l'IPSG, pour la recherche universitaire au Québec.

85 Université de Montréal_Institut pédagogique Saint-Georges (s.l., s.n., [1935]), Archives des FEC, chemise 500093.

86 "Vers le progrès — L'Institut pédagogique Saint-Georges ", L'enseignement primaire, 57, 1 (sept. 1935): $9-10$.

87 "Saint-Georges ", Les études, 3 (17 avr. 1939) :115-116.

88 Université de Montréal - Institut pédagogique Saint-Georges (s.1., s.n., [1935]), Archives des FEC.

89 Quelques faits qui attestent la valeur de la licence de l'Institut pédagogique Saint-Georges, Archives des FEC.

90 Cyrille (frère), Fédération des frères enseignants du Québec-Memento de ses principales activités : religieuses intellectuelles pédagogiques économiques pendant ses dix premières années (s.l., s.n., 1955), 8, Archives des FEC, chemise 2994.

91 Frère Chrysostome (Deshayes), La notion de relation chez l'enfant (1939) et Une étude critique du test d'intelligence logique de J.-M. Lahy (1939) // Frère Léo (Langlois), Adaptation à la Belgique des "Premiers tests de lecture" du professeur A.I. Gates (1939) et Test de tempérament et de caractère (1956) // Frère Luc (Brochu), La méthode de Rorschach appliquée à un groupe de délinquants et à un groupe contrôle (1942) // Frère Dominique (Gauthier), Les enfants déficients de l'oüe et leur développement mental et social (1946) // Frère Grégoire (Legault), La valeur du coloriage des croquis en pédagogie catéchistique (1950) // Frère Paul Griéger,: Étude pratique du caractère (1962) // Robert Tousignant sous le nom de frère Clément, Conception actuelle de l'inspection scolaire (1962).

92 Chrysostome (frère), "Morons ou génies ! ", L’hôpital, 2, 4 (mars 1938) : 185, Archives des FEC, chemise 500276. // Chrysostome (frère), "Ces princes héritiers du talent ! ", Propos scolaires - Congrès d'éducation de Hull (s.l., s.n., 1939) : 63-80, Archives des FEC, chemise 500276. // Chrysostome (frère), Le procès du quotient intellectuel - Extrait de la revue trimestrielle canadienne - juin 1940 (s.l., s.n., 1940), Archives des FEC, chemise 500276. 
93 Henrique Justo (irmao), Teste de inteligencia lógica elaborado no instituto de pedagogía S. Jorge (Mont De La Salle) — Universidade de Montréal - I: 9-12 anos, $4^{\mathrm{e}}$ ed. (Porto Alegre : Livraria S. Antonio, n.d) // Henrique Justo (irmao), Teste de inteligencia lógica elaborado no instituto de pedagogía S. Jorge (Mont De La Salle) —Universidade de Montréal-II: 13-18 anos, $7^{\mathrm{e}}$ ed. (s.l., s.n., n.d.) // Henrique Justo (irmao), Teste de atençao elaborado pelo instituto de pedagogía S. Jorge da universidade de Montréal, $12^{\mathrm{e}} \mathrm{ed}$. (Porto Alegre : Liv. S. Antonio, n.d) // Henrique Justo (irmao), «Teste do Instituto de Pedagogia S. Jorge da Universidade de Montréal ", Teste de carater a o alcance de todos,9e ed. (Porto Alegre : Livraria S. Antonio, 1966) // Henrique Justo (irmao), "Teste de atençao elaborado pelo Instituto de Pedagogia S. Jorge da Universidade de Montréal ", Testes psicológicos (Canoas : La Salle, 1996).

94 Lettre du frère Ephrem au frère Charles Mercier, Archives des FEC, chemise 4544.

95 Maurice-Alexandre (frère), Jean-Baptiste De La Salle, pionnier de l'éducation moderne, thèse de licence en pédagogie, École de pédagogie et d'orientation, Université Laval, 1953, 140. On en trouve une copie aux AFEC, chemise 514917.

96 Un demi-siècle au Mont-Saint-Louis-1888-1938-L'album jubilaire (Montréal : Imprimerie De-La-Salle, 1939), 571.

97 Société de pédagogie - Constitution, Archives des FEC, chemise 707.

$9810^{\circ}$ réunion annuelle - Programme-souvenir, La société de pédagogie de Montréal, 1947, Archives des FEC, chemise 510432. On a omis la conférence du frère Léo, auteur d'une grammaire : L'influence de la langue anglaise sur la langue française telle qu'elle est parlée et écrite dans la province de Québec, Archives des FEC, chemise 507906.

99 La société de pédagogie de Montréal_-Programme du vingt-cinquième anniversaire, (s.1. : s.n., 1963), Archives des FEC, chemise 500879.

100 Société de pédagogie de Montréal - Cotisations pour l'année 1939-1940, Archives des FEC, chemise 510440.

101 Dominique (frère), Accélération et enrichissement, coll. "Les conférences pédagogiques " vol. 1, no 5 (Montréal : Centre de psychologie et de pédagogie, 1944-45) //

Chrysostome (frère), Allons jusqu'aux faits (Montréal : La société de pédagogie de Montréal, 1940), Archives des FEC, chemise 500276. // Grégoire (frère), Les méthodes actives et l'enseignement du catéchisme, coll. "Les conférences pédagogiques » vol. 2, $\mathrm{n}^{\circ}$ 1 (Montréal : Centre de psychologie et de pédagogie, 1944-45) // Grégoire (frère), Coup de sonde dans les connaissances catéchétiques des élèves de sixième année, Bulletin $\mathrm{n}^{\circ}$ 6 (s.l. : La société de pédagogie de Montréal, n.d), Archives des FEC, chemise 500879. // Grégoire (frère), De l'utilisation des tests objectifs comme procédé de mémorisation et d'enseignement, Archives des FEC. // Ephrem (frère), Précis de statistique, Bulletin de la société de pédagogie de Montréal nos $15-16$ (Montréal, Le Centre de psychologie et de pédagogie, n.d.), Archives des FEC, chemise 500879.

102 Livres des procès-verbaux des réunions du Conseil et des assemblées générales de la Société de pédagogie de Montréal, Archives des FEC, chemise 510431.

103 Selon Nive Voisine (Les Frères des écoles chrétiennes au Canada-Tome III-Inquiétudes et renouvellement - 1946-1987 (Sainte-Foy : Anne Sigier, 1999), 162-163), en 1956, " la majorité des frères lasalliens enseignent désormais au secondaire".

104 Georges Croteau, Les frères éducateurs 1920-1975 - Promotion des études supérieures - Modernisation de l'enseignement public, (Montréal : HMH, 1996) et Paul-André Turcotte, L'enseignement secondaire public des Frères éducateurs, 19201970 (Montréal : Bellarmin, 1988) ont analysé ce phénomène pour l'ensemble des communautés enseignantes masculines.

105 Antonio Caporicci, "The Contribution of the Brothers of the Christian Schools to Education in Lower Canada, 1837-1847 ", mémoire de maîtrise, Université McGill, 1983, 76. 